\title{
Glassy Carbon: A Potential Dental Implant Material
}

\author{
E. E. HUCKE, R. A. FUYS, and R. G. CRAIG \\ Department of Materials and Metallurgy and School of Dentistry, \\ University of Michigan, Ann Arbor, Michigan
}

\begin{abstract}
Summary
The carbonization of certain polymers under carefully controlled conditions yields glassy carbon bodies which may have potential application in the dental profession. Considerable evidence is now available for the good compatibility of a variety of carbonaceous materials with tissue and bone. Many carbons remain relatively inert in the human environment compared to metals and polymers. Disordered carbons with strengths exceeding that of graphite are commercially available with elastic modulus in the same range as that reported for bone $(\sim 3 \times$ $10^{6} \mathrm{psi}$ ). The object of this study was to determine mechanical properties and characterize the structure of a new porous glassy carbon material.

Compressive and tensile strength data were obtained for carbon samples with a wide range of pore structures. A scanning electron microscope and a mercury porosimeter were used to observe variations in pore structures. The porous materials tested exhibited compressive strength values up to $48,000 \mathrm{psi}$ and ultimate tensile strength values up to $7,500 \mathrm{psi}$. Sonic modulus values from 1 to $4.6 \times 10^{6} \mathrm{psi}$ have been measured. Some carbon samples had interconnected porosity exceeding $50 \%$ and median pore diameters greater than $50 \mu$. Material with an interconnected mean pore size as small as $46 \AA$ has also been produced. Preliminary animal implant studies are in progress.
\end{abstract}

\section{INTRODUCTION}

A variety of studies of the behavior of carbon as a biomaterial has shown encouraging results. Several recent publications point out potential applications ranging from heart valves and pumps to prosthetic devices, including dental implants (1-4).

(C) 1973 by John Wiley \& Sons, Inc. 
The success of carbon appears to be associated with its chemical inertness in the body together with an attractive set of mechanical properties. While carbon of many varieties has been used, better results have been obtained with one or more of the forms which lack a high degree of crystallinity. Such materials have been called glassy, vitreous, or Pyrolite* carbons. The names applied stem more from the appearance of fracture surfaces, which resemble black glass, than from a knowledge of the atomic structure. In all instances, however, the structure is substantially different than graphite and results in general in a harder and stronger material. While the methods of preparation vary and the details of structure undoubtedly differ, these materials have many similar properties. All are apparently made by the controlled decomposition of a carbon containing molecular structure. In the case of Pyrolite, ${ }^{*}$ which is normally a thin coating on other materials, the molecular structure is formed from a gas phase which undergoes decomposition almost simultaneously with the deposition on the surface being coated in a fluidized bed. Under controlled conditions of temperature and composition of the gas phase, a strong, disordered, isotropic carbon is obtained which should not be confused with the highly anisotropic pyrolitic graphite made by similar techniques but with different deposition variables. The glassy carbons are prepared by careful decomposition of various crosslinked polymers. A wide variety of polymers will evidently produce glassy carbons, but most results have been obtained with either phenol formaldehyde or furfural-based polymers. In all cases it is possible to obtain, after heating to temperatures in the range of $1000-3000^{\circ} \mathrm{C}$, carbon of high purity with very little well-defined crystallinity. The exact structure of these carbons has not been unambiguously defined. It is well known, however, that small regions (on the order of $50 \AA$ develop a layered structure similar to graphite, but they still lack a well-defined crystal structure. In all instances a substantial number of voids is intimately included within the structure giving densities well below that of graphite. These voids are usually not interconnected, and therefore, a material is obtained that is impermeable to fluids.

* Registered trademark of Gulf Atomic Inc. 
In carbon for biomedical applications the chemical purity is undoubtedly an important factor, but the degree of atomic disorder appears also to be important since it governs the surface chemistry of the solids, and hence the potential interaction with any surrounding living tissue (4). The details of this interaction and the degree to which controlled variation is possible through control of carbon structure must await further research.

While all potential biomaterials must exhibit a high degree of biocompatibility, dental implants impose many additional property limitations. First, the nature of dental implants requires transmission of a complicated set of variable loads to the skeletal system. Not only does the implant material need to possess adequate strength and toughness, but it must transfer loads so that stress concentration at its interface with the body is minimized. Failure to achieve satisfactory transfer of the load will lead to resorption of bone. It has been noted that the problem of biocompatibility is much more severe when stresses are applied to material in vivo, particularly if there are shear strains at the implant interface $(2,5)$. In addition, a tooth implant must form a successful transcutaneous seal.

In all of the above respects, glassy carbon appears to offer substantial advantages over other available materials. Its basic biocompatibility in the body and the ability to form satisfactory transcutaneous passages in unstressed conditions has been demonstrated (1-4). While it is possible to aid in minimizing stress concentrations with clever design, the elastic modulus of other biocompatible materials, such as metals and various ceramics, is so much higher than bone that a satisfactory solution by design measures alone becomes difficult, if not impossible. It would be much more desirable to have an implant material with a modulus about equal or preferably less than that of the bone being adjoined. Unfortunately, most materials with a modulus below 2 to $3 \times 10^{6}$ psi (range of various bones) are either not strong enough or are not biocompatible. Figure 1 shows a plot of the ranges in compressive strength versus modulus for many candidate materials in comparison to various hard body tissues. In all instances, except for the metals, compressive strengths have been shown; for the metals, yield strength has been used. The other materials, including the carbons, are 


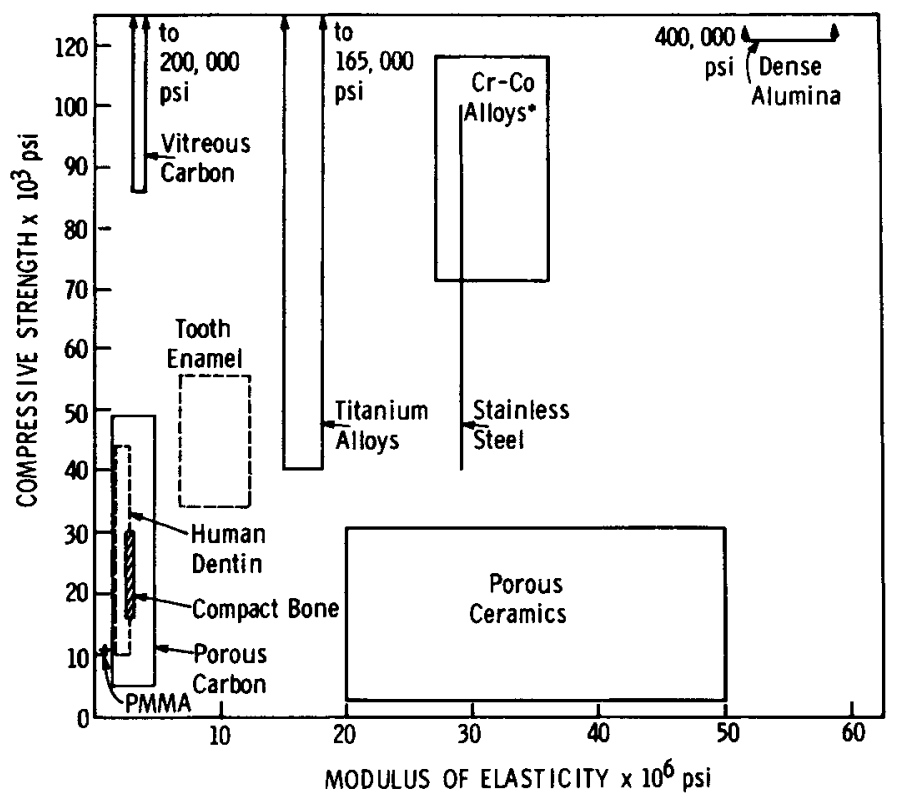

Fig. 1. Compressive strength vs. modulus of elasticity for various implant materials.

basically brittle, but fortunately the body's skeletal structure also is brittle so that careful emulation of loading conditions on this material should permit substitution of a brittle material. Figure 1 shows that various biocompatible metals and ceramics have more than adequate strength to match the hard body tissues, but both classes are far stiffer, and would therefore tend to cause larger strains in the supporting structure than in the implant under a given load. Only the carbons appear to have a modulus in the correct range while maintaining a useful strength level.

\section{POROUS GLASSY CARBONS}

In order to further lower the modulus, porous varieties of glassy carbon have been prepared at The University of Michigan by controlled pyrolysis of thermosetting polymers. These 
materials when made with various processing parameters yield samples with a wide range of pore size and total pore volume and a range of mechanical properties.

Typical structures of a coarse and a fine-pore material as revealed by scanning electron microscopy are shown in Fig. 2. Median interconnected pore sizes as small as $46 \mathrm{~A}$ and as large as $50 \mu$ have been produced. Interconnected pore volumes exceeding $50 \%$ have been achieved. Typical mercury intrusion porosimetry curves are shown in Fig. 3. Median pore diameters varied from 0.01 to $50 \mu$ and the interconnected porosity from about 0.2 to 1.2 $\mathrm{cc} / \mathrm{g}$.

Figure 4 shows porosimetry curves for two materials used in implant studies. One is a coarse-pore variety devised to allow tissue in-growth of the type noted by others in porous ceramics (6). Mechanical property data also are noted on this figure. In Fig. 5, a piece of this material has been fashioned into an alveolar ridge augmentation implant. This material, as well as the finer pore varieties, can be easily shaped with the usual dental grinding instruments. The finished piece can be polished with no tendency for particles to break out of the surface, even in the case of the coarse-pore material. Figure 6 shows samples made from the coarse-pore material for sub- and transcutaneous implants in rabbits.

The finer pore material can be polished to a mirror surface and appears solid to the unaided eye, as can be seen in Fig. 7. Still finer pored materials have been produced.

The mechanical property range thus far produced was shown in Fig. 1. The strengths obtainable, together with the lower modulus should yield at attractive material for dental implants. Since the mechanical properties depend on both the pore volume and size as well as the state of perfection of the carbon structure, tailoring of properties is possible. As yet insufficient data has been gathered to establish quantitatively the above property relationships, however, some typical values for compressive and tensile strength (dimentral-compression test) are shown in Table I. Representative hardness values for various samples processed differently together with those for commercially available materials are shown in Table II. Modulus of elasticity data obtained by sonic method are being gathered, with materials thus 
268 / HUCKE, FUYS, AND CRAIG

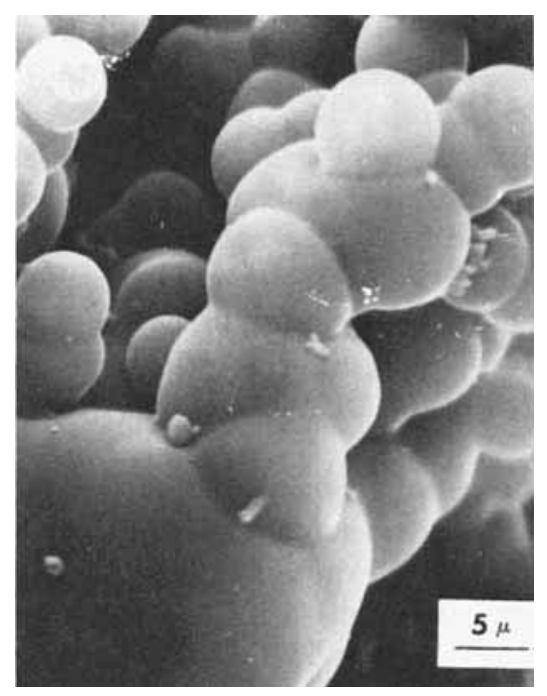

(a)

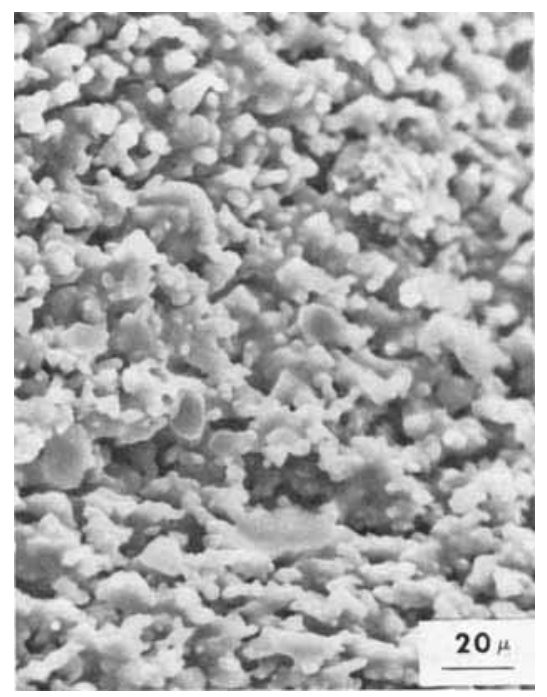

Fig. 2(b) (continued) 


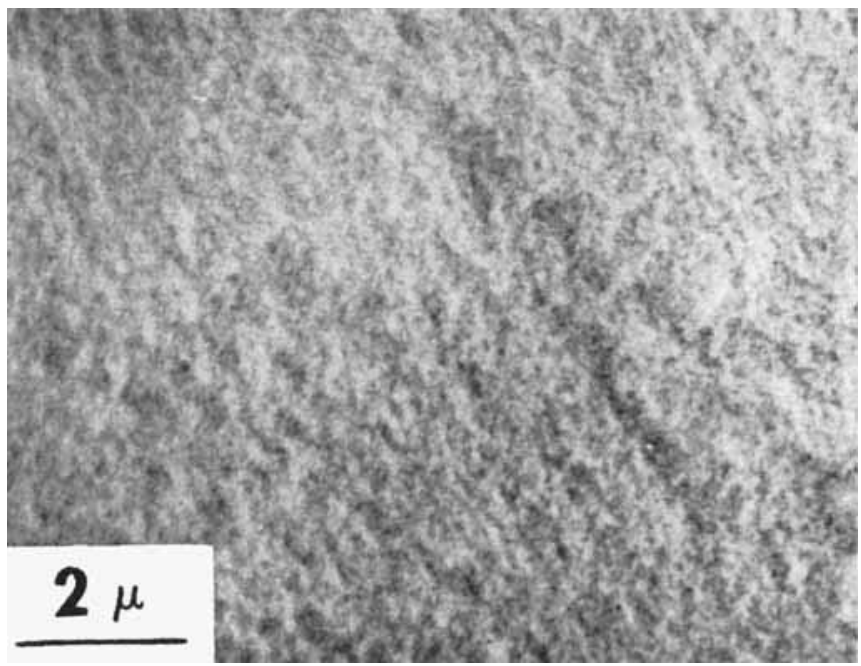

(c)

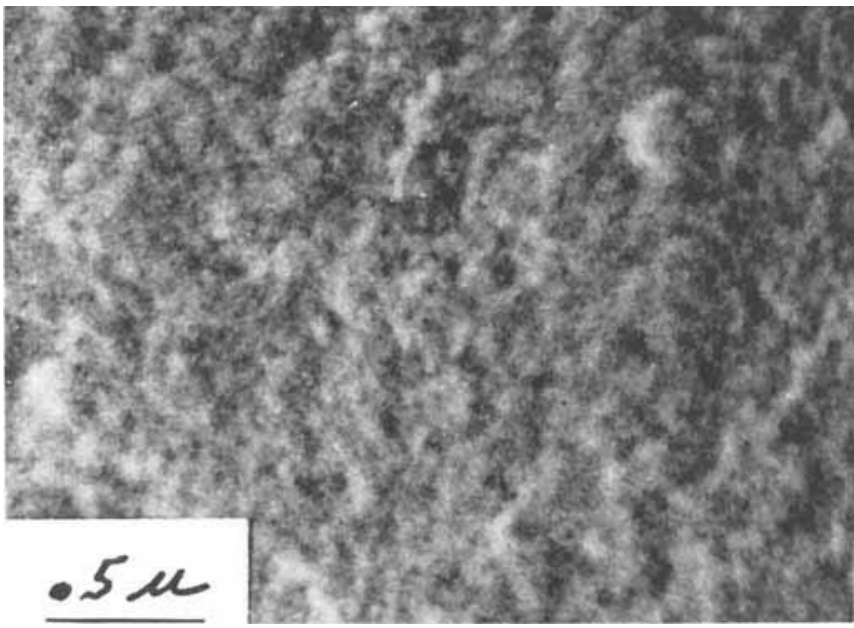

(d)

Fig. 2. Scanning electron micrographs of ( $a$ and $b$ ) coarse and (c and d) finepore glassy carbons. (a) Sample 315-40, (b) Sample 315-45A, (c and d) Sample $311-36$. 


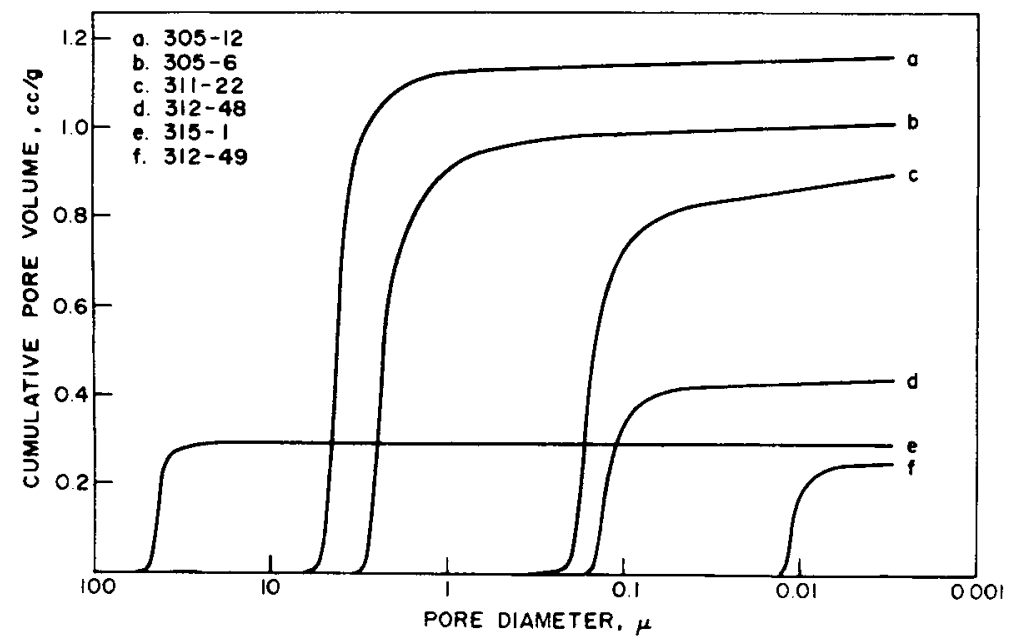

Fig. 3. Typical mercury porosity data for porous glassy carbons.

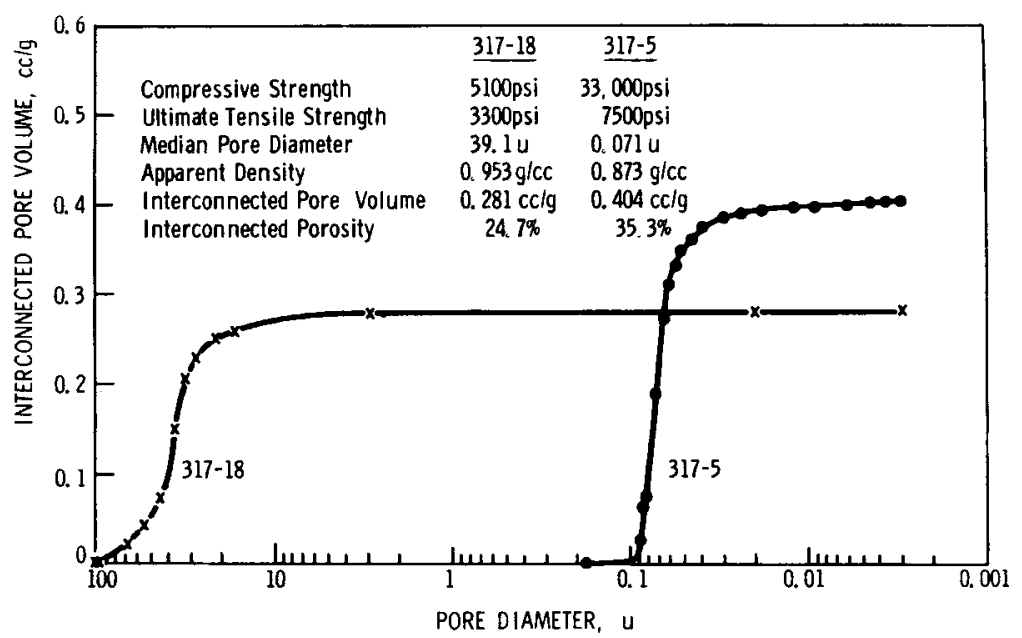

Fig. 4. Data for implant samples.

far examined exhibiting values from 1 to $4.6 \times 10^{6}$ psi. Still further variation should be achievable.

While all carbons are brittle, considerable difference in the energy required to fracture may exist depending on microstructure (4) and on degree of crystallinity. No conclusive 


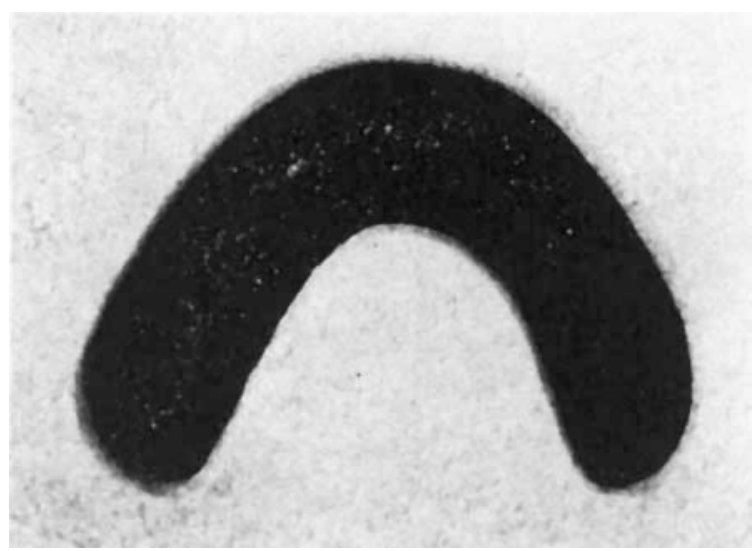

Fig. 5. Coarse-pore carbon for alveolar ridge augmentation.

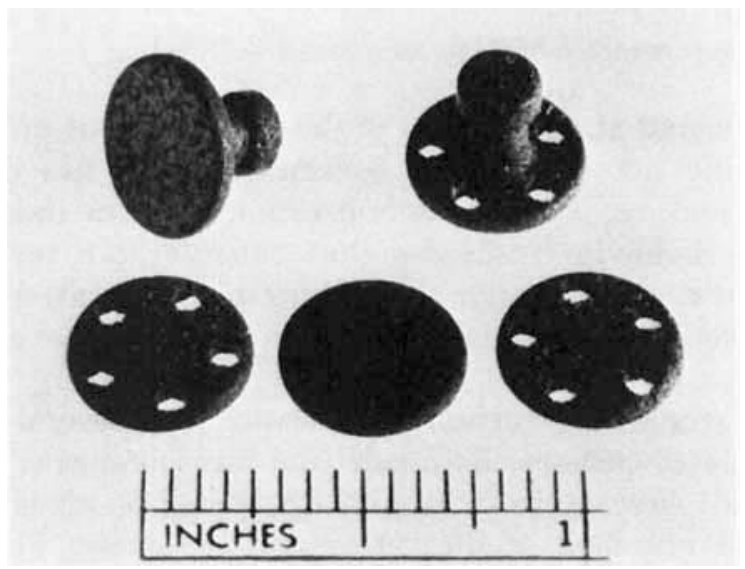

Fig. 6. Coarse-pore carbon implants from Sample 317-18.

evidence is yet compiled, however, early experiments on the porous material have indicated that the fine pores may act as crack arrestors. Mechanical tests show gradual failure with increasing loads rather than sudden catastropic fracture; this is somewhat similar to results reported on porous ceramics (6). In addition, Vickers hardness tests on porous samples have at times been carried up to $50 \mathrm{KG}$ without total failure of the sample. 


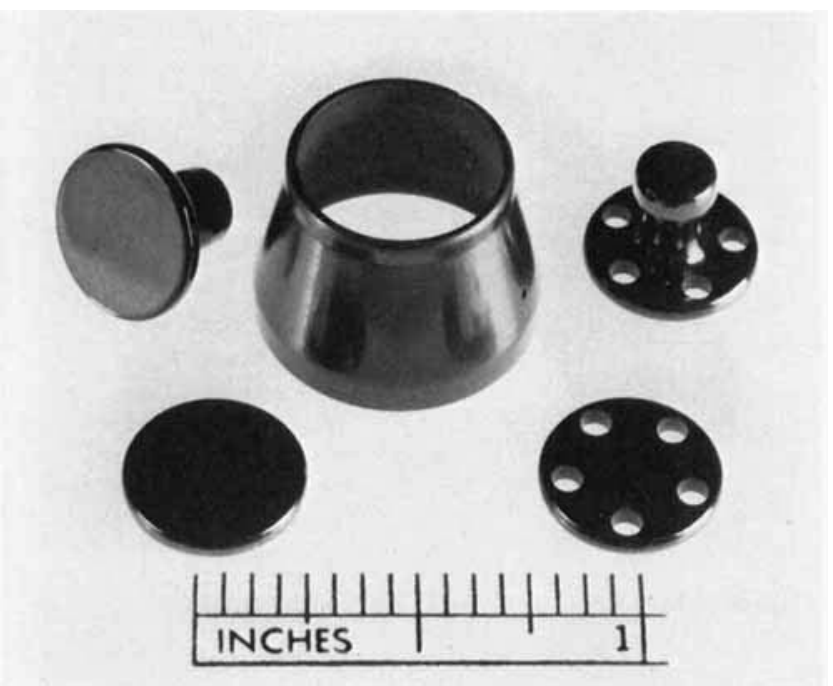

Fig. 7. Fine-pore carbon implants from Sample 317-5.

Cracks appeared at the corners of the impression at much lower loads but did not grow under constant load. It has also been noted that total recovery of the impression from the indenter can occur; this behavior indicates that considerable recoverable deformation can occur prior to fracture in these materials. Such behavior would be of real benefit in applications as dental implants.

Implant studies are currently underway with several varieties of porous glassy carbons. Both sub- and transcutaneous implants (nonstressed) were made in rabbits. Sacrifice was made after six months and histologic studies are now in progress. The results were clinically acceptable, tentatively confirming other studies showing excellent compatibility and ability to form transcutaneous seals. Further studies, including single tooth implants, are planned.

The authors would like to acknowledge support of ARPA Contract No. DAHC15-17-C-0283 and the United States Public Health Service General Research Support Grant RR05321-10 from the National Institute of Dental Research, National Institute of Health, Bethesda, Maryland, in the evaluation of the mechanical properties of many of the carbons studied. 
TABLE I

Typical Mechanical Property Data for Porous Glassy Carbons

\begin{tabular}{ccc}
\hline Sample & $\begin{array}{c}\text { Compressive strength } \\
(\mathrm{psi})\end{array}$ & $\begin{array}{c}\text { Ultimate tensile } \\
\text { strength }(\mathrm{psi})\end{array}$ \\
\hline $317-41 \mathrm{~A}$ & 7,400 & 1910 \\
$317-41$ & 10,000 & 2350 \\
$317-43$ & 15,000 & 2470 \\
$317-40$ & 27,100 & 4500 \\
$317-39$ & 30,700 & 5530 \\
$317-33$ & 40,200 & 5600 \\
\hline
\end{tabular}

TABLE II

Vickers Hardness Data for Porous Glassy Carbons

\begin{tabular}{lc}
\multicolumn{1}{c}{ Sample } & $\mathrm{DPH}^{a}$ \\
\hline $312-33,2000^{\circ} \mathrm{C}$ & 68 \\
$312-46,680^{\circ} \mathrm{C}$ & 100 \\
$312-46,2000^{\circ} \mathrm{C}$ & 106 \\
$312-45,680^{\circ} \mathrm{C}$ & 138 \\
$312-45 \mathrm{~A}, 2000^{\circ} \mathrm{C}$ & 152 \\
$315-46,2000^{\circ} \mathrm{C}$ & 240 \\
Commercially available glassy carbons & $171-312$ \\
\hline
\end{tabular}

a 3KG Load.

\section{References}

1. J. Benson, J. Biomed. Mater. Res., 5, (No. 2, Part 1), 41 (1971).

2. V. Mooney, P. K. Predicki, J. Renning, and J. Gray, J. Biomed. Mater. Res., 5, (No. 2, Part 1), 143 (1971).

3. J. A. von Fraunhofer, P. R. L'Estrange, A. O. Mack, Biomed. Eng., 114, (1971).

4. J. C. Bokros, E. Dalle-Molle, B. D. Epstein, F. J. Schoen, and D. P. Snowden, Biocompatibility of Carbon, Report PH43-67-1411-3, Gulf General Atomic Company, San Diego, California, Feb. 19, 1971.

5. J. Charnley, In Modern Trends in Biomechanics, Chapter 3, 1970, Butterworths. 
6. S. F. Hulbert, J. J. Klawitter and C. N. Wilson, Fabrication and Evaluation of Ceramic Implant Materials for Replacement of Teeth and Bone Mechanical Strength Testing of Highly Porous Calcium Aluminate Ceramics, Report No. NIH-70-2122, College of Engineering, Clemson University, Clemson, South Carolina, May 1971. 